\title{
The DNA Of Materials
}

Wathiq N. Abdul-Razzaq, West Virginia University, USA

Ryan K. Bushey, West Virginia University, USA

\begin{abstract}
Introductory physics labs provide many challenging yet applicable experiments and concepts to the many fields of engineering. One such lab has been developed at West Virginia University that explores resistivities of several different materials and ties this concept into electrical engineering practices and standards. Many students do not realize that resistivity is a very significant quantity that contributes greatly to the understanding of natural as well as man-made materials; it is the DNA of materials. This lab provides engineering students an opportunity to not only learn physics of materials, but to also gain real-world experience and understanding of why certain materials are used in electronic devices and when designing and constructing buildings. This lab has been implemented in several introductory lab levels here at West Virginia University, and all have been met with enthusiasm and strong participation. In our pre-engineering physics course, this has been especially effective, as it has given the students some hands-on experience with physics principles that they will make use of in their future careers.
\end{abstract}

\section{INTRODUCTION}



he applicability of materials in the use of electrical circuits has been standardized by the National Fire Protection Association that publishes the National Electrical Code. ${ }^{1}$ This code provides building contractors, electrical engineers, and electricians the rules that need to be followed to properly install electrical wiring. This code requires the use of specific materials, lengths, cross-sectional areas, (otherwise known as the gauge of the wire), and several other factors in order to provide a safe installation of the electrical framework.

The students are probably aware that different materials consist of different atoms. However, because of these differences in the atoms of materials and how the atoms are arranged in the material, the ability to pass electric current varies from material to material. In other words, each material resists electric current differently than any other material. This unique property of materials is called the resistivity $\rho$.

Materials are characterized as metals, semiconductors, insulators or superconductors based on the magnitude and the behavior of their resistivity. Metals are excellent conductors used in conducting electricity from one place to another, while semiconductors are widely used in making computer's chips. Insulators, such as rubber, are used to protect us from electrocution. Superconductors have zero resistivity; electric current could pass without loss through these materials. A sudden change in the behavior of resistivity with temperature could indicate a transition from one state of the material to another state of different properties.

\section{PROCEDURE}

In this activity, students will identify four "unknown" materials by finding their resistivities and comparing their values to those given in Table 1. 
Table 1: Resistivity, $\rho$, of different materials, at $20^{\circ} \mathrm{C}$. Each material has its own unique resistivity value.

\begin{tabular}{|c|c|c|}
\hline Material (metal) & \multicolumn{2}{|c|}{$\begin{array}{l}\text { Resistivity } \rho \\
\quad(\text { ohm m) }\end{array}$} \\
\hline Silver (metal) & 1.59 & $\mathrm{x} 10^{-8}$ \\
\hline Copper (metal) & 1.68 & $\mathrm{x} 10^{-8}$ \\
\hline Aluminum (metal) & 2.65 & $\mathrm{x} 10^{-8}$ \\
\hline Tungsten (metal) & 5.6 & $\mathrm{x} 10^{-8}$ \\
\hline Iron (metal) & 9.71 & $\mathrm{x} 10^{-8}$ \\
\hline Platinum (metal) & 10.6 & $\mathrm{x} 10^{-8}$ \\
\hline Manganin (metal) & 48.2 & $\mathrm{x} 10^{-8}$ \\
\hline Lead (metal) & 22 & $\mathrm{x} 10^{-8}$ \\
\hline Mercury (metal) & 98 & $\mathrm{x} 10^{-8}$ \\
\hline Nichrome:Ni,Fe,Cr (metallic alloy) & 100 & $\mathrm{x} 10^{-8}$ \\
\hline Constantan (metal) & 49 & $\mathrm{x} 10^{-8}$ \\
\hline Carbon (graphite) (semiconductor) & $3-60$ & $\mathrm{x} 10^{-5}$ \\
\hline Germanium (semiconductor) & $1-500$ & $\mathrm{x} 10^{-3}$ \\
\hline Silicon (semiconductor) & $0.1-60$ & $\mathrm{x} 1$ \\
\hline Glass (insulator) & $1-10000$ & $\mathrm{x} 10^{9}$ \\
\hline Quartz, fused (insulator) & 7.5 & $\mathrm{x} 10^{17}$ \\
\hline Hard rubber (insulator) & $1-100$ & $\mathrm{x} 10^{13}$ \\
\hline
\end{tabular}

The following describes the lab that has been introduced and discusses the steps through which the students proceed. We provide an introduction that establishes the principles behind the lab.

The total resistance, $\mathrm{R}$, to current of any material depends not only on the nature of the atoms of the material (resistivity, $\rho$ ), but also on the length, L, and the cross sectional area, A, of the material, Figure 1 . R would be expected to be greater for a longer material or less for larger cross sectional area of the material. Experimentally, the dependence of $\mathrm{R}$ on $\rho, \mathrm{L}$ and $\mathrm{A}$ can be expressed as

$$
\mathrm{R}=\rho \mathrm{L} / \mathrm{A} \text {. }
$$

(Equation 1)

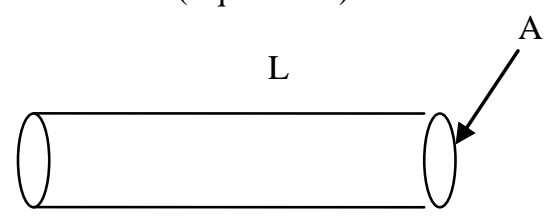

Figure 1: Resistance depends on the length $L$ and the cross sectional area $A$ of the material beside the resistivity.

The inverse of resistivity $(1 / \rho)$ is called conductivity. The higher the resistivity, the less conductive the material would be to electric current.

The students then proceed with the following measurements. They are provided with two multimeters, a battery, four spools of unknown material, and some alligator clips to make the connections. The multimeter can be 
used as a voltmeter $\mathrm{V}$, an ammeter $\mathrm{A}$ or an ohmmeter $\Omega$. They cut an approximately $20 \mathrm{~cm}$ length of the unknown material (in the form of a string) on spool \#1. Connect this material $\left(\mathrm{R}_{1}\right)$ with a battery, an ammeter A and a voltmeter $\mathrm{V}$ as shown in Figure 2.

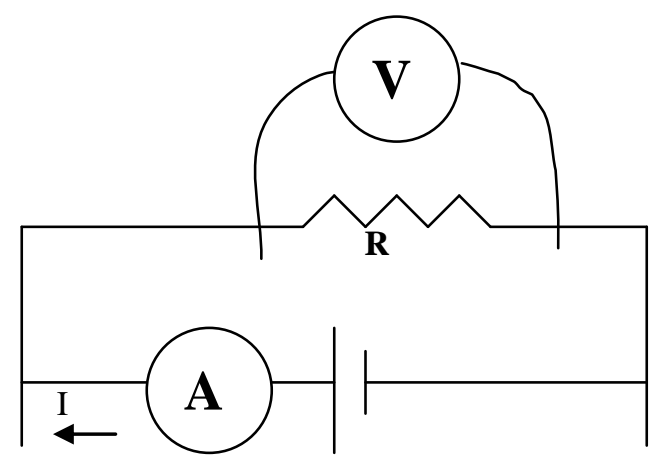

Figure 2: The circuit connection, $R$ represents the material, $V$ is voltmeter, $A$ is the Ammeter.

Using Ohm's Law, $\mathrm{R}=\mathrm{V} / \mathrm{I}$, the students calculate the resistance $\mathrm{R}$, where $\mathrm{V}$ is the voltmeter reading and $\mathrm{I}$ is the ammeter reading. They then use the multimeter as an ohmmeter by connecting the two leads of the multimeter to the material $R$. They should then compare this measured value of $R$ with the value for $R$ that they calculated previously. Then, they measure the length of the material, $\mathrm{L}$, and determine the cross sectional area of the material. Using these measurements and the resistance, R, they find the resistivity using Equation 1. At this point, the students should try to identify the material by referring to Table 1 . Repeat for the materials on spools 2, 3, and 4 .

If they are getting the idea that only wires have resistance or can allow current to pass through them, then they should try something different: use the ohmmeter function of the multimeter to measure the resistance between two points in their body. One suggestion: touch one lead with a finger of the right hand and the other lead with a finger of the left hand. Can they determine the resistivity of their body? To understand further, we pose the question of what factors make this rather difficult. Another brief exploratory procedure is to have the students use the ohmmeter function to measure the resistance of two lights bulbs of different wattage.

\section{RESISTIVITY AND MAGNETISM AT NANO-IEVEL: THE SUBJECT OF A NOBLE PRIZE}

After students understand how to identify materials based on the magnitude of the resistivity, they, with the help of the lab's Teaching Assistant, discuss the 2007 Nobel prize in physics that was awarded for work based on the behavior of resistivity in the presence of magnetic field, namely magnetoresistance.

Magnetoresistance (MR) is the change of resistivity of a conductor when it is placed in an external magnetic field. For ferromagnets like iron, cobalt and nickel this property will also depend on the direction of the external field relative to the direction of the electric current ${ }^{2}$. Exactly 150 years ago W. Thomson ${ }^{3}$ measured the behavior of the resististivity of iron and nickel in the presence of a magnetic field. He wrote "I found that iron, when subjected to a magnetic force, acquires an increase of resistance to the conduction of electricity along, and a diminution of resistance to the conduction of electricity across, the lines of magnetization". This difference in resistance between the parallel and perpendicular case is called anisotropic magnetoresistance (AMR) ${ }^{4}$. In general magnetoresistance effects are very small, at most of the order of a few per cent. The MR effect has been of substantial importance technologically, especially in connection with readout heads for magnetic disks and as sensors of magnetic fields. The most useful material has been an alloy between iron and nickel, $\mathrm{Fe}_{20} \mathrm{Ni}_{80}$ (permalloy). In general, however, there was hardly any improvement of the performance of magnetoresistive materials since the work of Thomson.

It was a great surprise when in 1988 two research groups independently discovered materials showing a very large magnetoresistance, now known as giant magnetoresistance (GMR). These materials are so called magnetic multilayers, where layers of ferromagnetic and non-magnetic metals are stacked on each other. The widths of the individual layers are of nanometre size - i.e. only a few atomic layers thick. In the original 
experiments leading to the discovery of GMR one group, led by Grünberg ${ }^{5}$, used a trilayer system $\mathrm{Fe} / \mathrm{Cr} / \mathrm{Fe}$, while the other group, led by Fert ${ }^{6}$, used multilayers of the form $(\mathrm{Fe} / \mathrm{Cr}) \mathrm{n}$ where $\mathrm{n}$ could be as high as 60 , figure 3 .

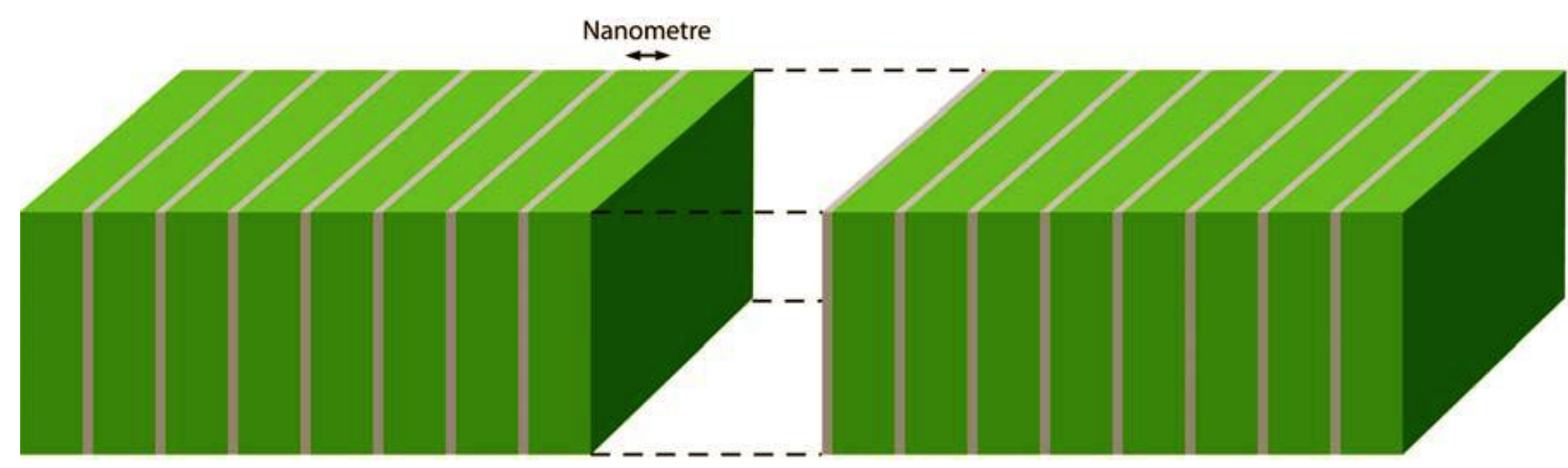

Figure 3. Schematic figure of magnetic multilayers. Nanometre thick layers of iron (green) are separated by nanometre thick spacer layers of a second metal (for example chromium or copper). The multilayer (Fe/Cr)n, with $\mathbf{n}$ as high as 60 , used by Fert's group".

This system that has large stacks of layers, show a decrease of resistance by almost $50 \%$ at $4.2 \mathrm{k}$ when subjected to a magnetic field. The $50 \%$ is a gaint decrease in comparison with few percentage decrease in alloys. Not only did Fert and Grünberg measure strongly enhanced magnetoresistivities, but they also identified these observations as a new phenomenon, where the origin of the magnetoresistance was of a totally new type. The discovery of giant magnetoresistance immediately opened the door to a wealth of new scientific and technological possibilities, including a tremendous influence on the technique of data storage and magnetic sensors. Thousands of scientists all around the world are today working on magnetoelectronic phenomena and their exploration.

An electrical current of electrons sent through a metallic system will always experience a resistance $\mathrm{R}$ that depends on the resistivity. There are a number of reasons for this. In a crystal the atoms will always vibrate (phonons) around their equilibrium positions, thereby deviating from the perfect lattice positions and the conduction electrons may be scattered by these deviations (electron - phonon interaction). Other important contributions to the resistance of a metal are scattering of electrons against impurities and defects. For paramagnetic metals there is no difference between the spin up and spin down electrons, and they contribute equally to the resistance. The Spin, the internal "rotation" of the electron, creates a magnetic moment. Each electron behaves like a small magnet, pointing either upwards or downwards. However, the resistance for the spin up or down electrons through the ferromagnetic (FM) layer and the scattering at the interface to the non-magnetic (NM) layer will be different called $\mathrm{R} \uparrow$ and $\mathrm{R} \downarrow$. Thus when the two FM layers (separated by a NM layer) have parallel spin polarizations (magnetizations), i.e. in the presence of an external magnetic field $(\mathrm{H})$, the resistance for the spin up channel is $2 \mathrm{R} \uparrow$, and for the spin down channel it is $2 \mathrm{R} \downarrow$. Standard addition of resistances for a parallel current configuration gives the following total resistance, $R_{H}$, in the presence of an external magnetic field; $R_{H}=2 R \uparrow R \downarrow /(R \uparrow+R \downarrow)$.

In the case of no external magnetic field, $(\mathrm{H}=0)$, the configuration between the two magnetic layers is antiparallel. However, when a spin up electron enters into the second FM layer it finds itself in a totally upsidedown situation. Thus the spin up particle will now experience a total resistance of $\mathrm{R} \uparrow+\mathrm{R} \downarrow$. The spin down particle will be affected in the same (but opposite) way and its resistance will be $\mathrm{R} \downarrow+\mathrm{R} \uparrow$. The total resistance will accordingly be $\mathrm{R}_{0}=(1 / 2)(\mathrm{R} \uparrow+\mathrm{R} \downarrow)$. Thus the difference in resistance between the two cases (magnetic field or not) becomes:

$$
\Delta \mathrm{R}=\mathrm{R}_{\mathrm{H}}-\mathrm{R}_{0}=-(1 / 2)(\mathrm{R} \uparrow-\mathrm{R} \downarrow)^{2} /(\mathrm{R} \uparrow+\mathrm{R} \downarrow)
$$

Thus the larger the difference between $\mathrm{R} \uparrow$ and $\mathrm{R} \downarrow$ the larger the negative magnetoresistance. This expression clearly shows that the magnetoresistance effect arises from the difference between the resistance behavior of the spin up and down electrons. 
We are in the process of having students measure magnetoresistance in the labs. We are also planning to have computer hard disks and readout heads available to the students to explore. This will serve as a reminder that the effect of magnetic field on resistivity is vital for compact hard disks. A small magnetic change can make a major difference in resistivity and thus to an electric current. This is the basis of a responsive sensor that uses giant magnetoresistance. Information is stored on hard disks in the form of small magnetic fields. As hard disks become smaller and more compact, more sensitive readout heads are required that can register small changes in magnetism. A read-out head based on giant magnetoresistance can be compared to a jet plane flying at about 19,000 miles/ hour one meter above the ground recording each blade of grass.

\section{CONCLUSION}

Resistivity is a very important concept not realized by the majority of the students. Materials are characterized as metals, semiconductors, insulators or Giant-magneto resistance based on the magnitude and the behavior of their resistivity. Pre-engineering students in particular need to understand the depth of resistivity. This lab attracts the students' attention to this concept.

Students were alerted to the fact that the 2007 Nobel Prize in physics was based on the concept of resistivity. The effect of magnetic field on the resistivity led to the discovery of giant magnetoresistance (GMR). This discovery opened the door to a new field of science, magnetoelectronics (or spintronics), where two fundamental properties of the electron, namely its charge and its spin, are manipulated simultaneously ${ }^{2}$. Emerging nanotechnology was an original prerequisite for the discovery of GMR, now magnetoelectronics is in its turn a driving force for new applications of nanotechnology. Thanks to this technique it has been possible to continue the miniaturization of hard disks, a necessity for many of the IT devices nowadays taken for granted: small but powerful laptops, search engines and music players with large memories.

\section{AUTHOR INFORMATION}

Dr. Abdul-Razzaq received his $\mathrm{PhD}$ in 1986 from the University of Illinois at Chicago. He completed his postdoctorate work at Michigan State University. Currently he is Professor of Physics and Director of Introductory Physics Curriculum at West Virginia University. In recent years he has had research activities in diverse areas including studies of magnetic and transport properties of thin films and multilayers, studies of magnetic nanoparticles, and applied studies related to health and environment. In addition, he does research in education.

Mr. Bushey received his Bachelor of Science in Physics from the University of Vermont in 2001, where his course work focus was in biophysics, specifically protein dynamics. Upon graduation he became a lead software engineer and then decided to pursue a graduate degree in order to become a high school physics teacher. He assisted Dr. Abdul-Razzaq in performing research in education as well as electromagnetic radiation. He has also taught biology and physics laboratories at West Virginia University. He is currently a high school teacher in Vermont.

\section{REFERENCES}

\footnotetext{
${ }^{1}$ National Fire Protection Agency (2007). NFPA Codes and Standards. Retrieved May $7^{\text {th }}, 2007$ from NFPA Codes and Standards Web Site: http://www.nfpa.org/categoryList.asp?categoryID=124\&URL=Codes\%20and\%20Standards

${ }^{2}$ The Discovery of Giant Magnetoresistance compiled by the Royal Swedish Academy of Sciences (2007).

${ }^{3}$ W. Thomson, "On the Electro-Dynamic Qualities of Metals: Effects of Magnetization on the Electric Conductivity of Nickel and of Iron", Proceedings of the Royal Society of London, 8, pp. 546-550 (1856-1857).

${ }^{4}$ I.A. Campbell and A. Fert, "Transport Properties of Ferromagnets" in Ferromagnetic Materials, ed. E.P. Wohlfarth, NorthHolland, Amsterdam, Vol. 3, p. 747 (1982).

${ }^{5}$ G. Binasch, P. Grünberg, F. Saurenbach, and W. Zinn, "Enhanced magnetoresistance in layered magnetic structures with antiferromagnetic interlayer exchange”, Phys. Rev. B 39, 4828 (1989).

${ }^{6}$ M.N. Baibich, J.M. Broto, A. Fert, F. Nguyen van Dau, F. Petroff, P. Eitenne, G. Creuzet, A. Friederich, and J. Chazelas, "Giant Magnetoresistance of (001)Fe/(001)Cr Magnetic Superlattices”, Phys. Rev. Lett. 61, 2472 (1988).
} 


\section{NOTES}

